\title{
Pulmonary tuberculosis in a male with sarcoidosis
}

\section{Marcin Skowroński, Anna Halicka, Aleksander Barinow-Wojewódzki}

Wielkopolska Centre of Pulmonology and Thoracic Surgery, Poznań, Poland

A 45-year-old nonsmoking male patient was admitted with a nonproductive cough. He was in the follow-up for sarcoidosis stage II for 2 years. This was based on computed tomography findings of enlarged mediastinal lymph nodes and multiple small scattered nodules. Fine-needle aspiration of the left paratracheal lymph node revealed epithelioid histiocytes and multi-nucleated giants cells. Smear and culture for acid-fast bacilli (AFB) of bronchial washings and lymph node aspirate were negative. As the patient was asymptomatic with normal respiratory function tests, he was under observation.

On the current admission, the physical examination was unremarkable. The erythrocytes sedimentation rate was $8 \mathrm{~mm}$ /hour. There was a very large number of parenchymal nodules mainly in the upper and perihilar zones in his chest X-ray (CXR), with parenchymal fibrotic changes (Figure 1). Smear for AFB of bronchial washings was negative. After 8 weeks, the culture on Löwenstein-Jensen medium of bronchial washings was positive for AFB. Anti-tuberculous treatment (ATT) was started. Drug susceptibility testing showed sensitivity to all first-line drugs. He tolerated the ATT well. There was no improvement on radiological imaging. He was discharged and treated as an out-patient in a continuation phase. The smear-negative samples after 2 and 6 months were culture negative. The CXR after the completion of the ATT did not show an improvement. He was seen in the out-patient department till the completion of the ATT. Then he was lost for further follow-up.

Sarcoidosis is recognized as noncaseating granulomas in contrary to caseating granulomas in tuberculosis (TB). Mycobacterium tuberculosis (MTB) may be an etiological agent in sarcoidosis [1]. The annual incidence of sarcoidosis varies from 0.73 per 1000000 in Japan to 71/100 000 in African-American females [1]. In 2017, there were fewer than 10 new TB cases per 100000 population in high-income countries, 150-400 in high TB burden countries, and above 500 in countries including Mozambique, the Philippines and South Africa [2].

In an Indian prospective study, $48 \%$ of all sarcoidosis patients had mycobacterial DNA and that was higher $(\mathrm{p}<0.001)$ compared to the controls [1]. However, the study was performed in a high TB burden country.

$3 \%$ of cases of parenchymal nodules and masses in sarcoidosis show cavitation [3]. A case of primary cavitary sarcoidosis which was initially treated as TB was reported [3]. In order to differentiate sarcoidosis from TB, endobronchial ultrasonography may help. Heterogeneous echotexture (53.4\% vs $12.6 \%$ ) and coagulation necrosis (26.1\% vs 3.3\%) were more frequent in TB lymph nodes [4].

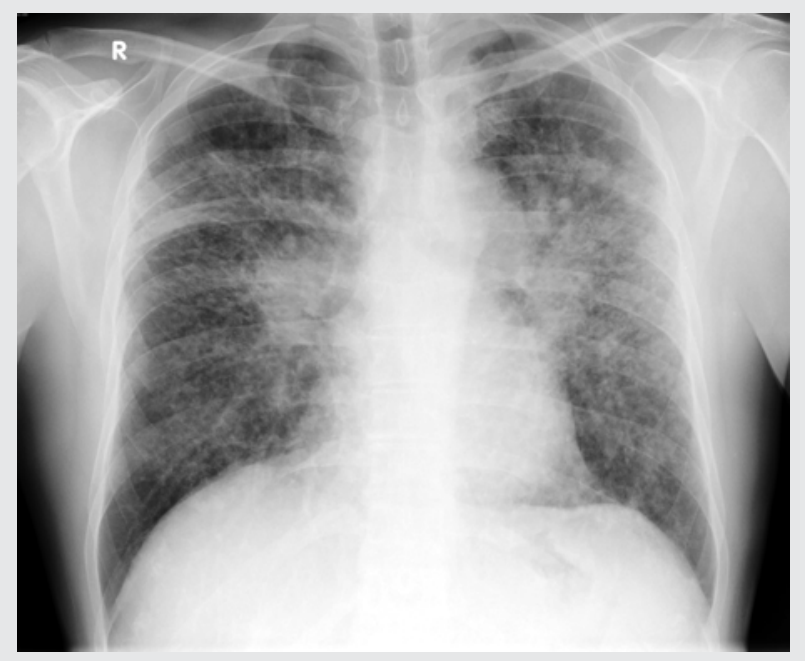

Figure 1. Diffuse bilateral and ill-defined interstitial nodules in the upper lobes and perihilar regions. Bilateral symmetrical hilar lymphadenopathy

Address for correspondence: Marcin Skowroński, Wielkopolskie Centrum Pulmonologii i Torakochirurgii, Ludwikowo k. Poznania, 62-050 Mosina; e-mail: marcinas@gazeta.pl DOI: 10.5603/ARM.2019.0043

Received: 11.05.2019

Copyright (C) 2019 PTChP

ISSN 2451-4934

Conflict of interest: none declared 
The socio-demographic features of these patients were explored. People with sarcoidosis were older, better educated, had a higher BMI and socioeconomic status [5]. In contrast, tobacco smoking and biomass for cooking were more frequent in TB.

Mycobacterial antigens may be responsible for initiating granulomas in sarcoidosis. Negative results of the histological stains or cultures may indicate that tests are not sensitive enough to detect these antigens. Apparently, TB and sarcoidosis may be a spectrum of the same disease [1]. The difference in the immune response of the host may result in a varying degree of presentation.

Though our patient presented first with sarcoidosis, later he developed TB. So the mycobacterial antigens might have not been detected at first. It is crucial to remember about this association as the misdiagnosis of TB may cause the spread of the disease with the immunosuppressive therapy.

\section{References:}

1. Mootha VK, Agarwal R, Aggarwal AN, et al. The sarcoid-tuberculosis link: evidence from a high TB prevalence country. J Infect. 2010; 60(6): 501-503, doi: 10.1016/j.jinf.2010.03.010, indexed in Pubmed: 20346973.

2. Global tuberculosis report 2018. Geneva: World Health Organization; 2018. Licence: CC BY-NC-SA 3.0 IGO.

3. Handa A, Dhooria S, Sehgal IS, et al. Primary cavitary sarcoidosis: A case report, systematic review, and proposal of new diagnostic criteria. Lung India. 2018; 35(1): 41-46, doi: 10.4103/lungindia.lungindia_225_17, indexed in Pubmed: 29319033.

4. Dhooria S, Agarwal R, Aggarwal AN, et al. Differentiating tuberculosis from sarcoidosis by sonographic characteristics of lymph nodes on endobronchial ultrasonography: a study of 165 patients. J Thorac Cardiovasc Surg. 2014; 148(2): 662-667, doi: 10.1016/j. jtcvs.2014.01.028, indexed in Pubmed: 24534680.

5. Gupta D, Vinay N, Agarwal R, et al. Socio-demographic profile of patients with sarcoidosis vis-à-vis tuberculosis. Sarcoidosis Vasc Diffuse Lung Dis. 2013; 30(3): 186-193, indexed in Pubmed: 24284291. 